\title{
Attention and olfactory consciousness
}

\author{
Andreas Keller* \\ Department of Philosophy, Graduate Center, City University of New York, New York, NY, USA
}

\author{
Edited by: \\ Naotsugu Tsuchiya, RIKEN, Japan \\ Reviewed by: \\ David Rosenthal, City University of \\ New York, USA \\ Giorgio Marchetti, Mind, \\ Consciousness, and Language, Italy \\ *Correspondence: \\ Andreas Keller, Department of \\ Philosophy, Graduate Center, City \\ University of New York, 365 Fifth \\ Avenue, New York, NY 10016, USA \\ e-mail: andreaskeller@runbox.com
}

Understanding the relation between attention and consciousness is an important part of our understanding of consciousness. Attention, unlike consciousness, can be systematically manipulated in psychophysical experiments and a law-like relation between attention and consciousness is waiting to be discovered. Most attempts to discover the nature of this relation are focused on a special type of attention: spatial visual attention. In this review I want to introduce another type of attention to the discussion: attention to the olfactory modality. I will first clarify the position of attention to smells in a general taxonomy of attention. I will then review the mechanisms and neuroanatomy of attention and consciousness in the olfactory system before using the newly introduced system to provide evidence that attention is necessary for consciousness.

Keywords: olfaction, attention, consciousness, odor, orbitofrontal cortex, piriform cortex

\section{INTRODUCTION}

Knowledge about the relation between attention and consciousness is invaluable for the empirical study of consciousness because attention can be varied experimentally and its effect on conscious perception can be measured. The discussion of how attention relates to consciousness has so far been based primarily on empirical results from the study of spatial visual attention (Dehaene et al., 2006; De Brigard and Prinz, 2010; Prinz, 2010; Van Boxtel et al., 2010). The goal of this paper is to introduce attentional shifts toward the olfactory modality as a second system in which attention and consciousness interact. The advantage of opening up the discussion to facts from a second type of attention is that it reduces the likelihood of mistaking special adaptations for general principles.

The main motivation to study attention in olfaction is the computational and anatomical simplicity of the olfactory system (Haberly, 2001; Lledo et al., 2005; Isaacson, 2010; Sela and Sobel, 2010). The study of simple systems to reveal general principles has contributed significantly to our understanding of many biological processes and the olfactory system is an ideal model system for consciousness research. It may even represent the minimal neuroanatomy that is required for conscious processing (Morsella et al., 2010).

Toward the goal of introducing attentional shifts toward olfaction as a system for the study of attention, this paper has three aims. The first aim is to develop a taxonomy of olfactory attention that is integrated into a general taxonomy of attention (Chun et al., 2011). The second aim is to review what is known about the brain structures that are involved in attentional shifts toward the olfactory modality and in the conscious processing of olfactory information. These first two parts together introduce a system in which questions about the relation between attention and consciousness can be studied. The third aim is to demonstrate the utility of the newly introduced system by answering one of these questions: is attention to smells necessary for the conscious processing of smells?

\section{A TAXONOMY OF OLFACTORY ATTENTION}

The study of attention in the olfactory modality is in its infancy. A search of literature databases will reveal that there are almost a thousand times more papers on visual attention than on olfactory attention. Before discussing attention in the olfactory modality it is therefore necessary to clarify what types of olfactory attention there are and how they relate to other types of attention. I will accomplish this by placing olfactory attentional processes into a recently introduced general taxonomy of attention (Chun et al., 2011).

The taxonomy proposed by Chun et al. (2011) is based on what type of information attention operates over. The most basic distinction is between external and internal attention. Internal attention operates over internally generated information such as memories, thoughts, or task rules. External attention operates over sensory information. The types of olfactory attention discussed in this paper are types of external attention. External attention can be further subdivided into attention to space, time, objects, or features of objects. In addition, there is attention to information generated by a specific modality. I will now discuss these five types of external attention (spatial attention, temporal attention, attention to objects, attention to features, and attention to a modality) in olfaction.

\section{SPATIAL OLFACTORY ATTENTION}

The paradigmatic case of spatial attention is the allocation of visual attention to positions in visual space. Visual spatial attention can be shifted overtly by body, head, and eye movements or covertly by computational means while the fixation point is unchanged. In every-day experience overt shifts of spatial visual attention are more prominent but covert shifts are also significant (for example, Wojciulik et al., 1998). Other senses with spatially structured phenomenology, like passive touch, also have computational mechanisms for covert spatial attention (Forster and Eimer, 2004).

Overt spatial shifts of attention are possible in olfaction. In the same way in which visual attention can be shifted in space by 
turning the head, olfactory attention can be shifted in space by placing the nose over different objects that are giving off odors. These overt shifts of olfactory spatial attention allow us to locate sources of odors and to track scents (Porter et al., 2006). The study of overt shifts of spatial attention to elucidate the relation between attention and consciousness is complicated by the fact that overtly shifting olfactory or visual attention changes the sensory input. Covert shifts, in which the sensory input remains the same and the only difference is how attention is distributed over the visual field, have been more informative for questions about consciousness and attention.

However, covert spatial shifts of attention are only possible in modalities in which the sensory experience has a spatial structure. Odor experiences are one example of spatially unstructured sensory experiences (Radil and Wysocki, 1998; Frasnelli et al., 2008). Some researchers have suggested that an olfactory experience has a very simple spatial structure that consists of two locations: left (nostril) and right (nostril; Porter et al., 2005, 2006). Even if this is the spatial structure of an olfactory experience, there is no evidence that attention can be shifted between the nostrils. There is therefore no evidence for covert spatial olfactory attention.

\section{TEMPORAL OLFACTORY ATTENTION}

It has been shown that in vision attention can not only be allocated over space but also over time (Coull and Nobre, 1998). This type of attention can be tested in experiments which involve a cue that informs the subject at what time after the cue a stimulus can be expected. The subject will then allocate attention toward the time window in which the stimulus is expected. Allocating attention temporally is also possible in olfaction. It is, for example, possible to pay attention to odors only on Wednesdays, but not on the other days of the week. However, olfactory experiences have a peculiar temporal structure which makes the fast attentional shifts that are characteristic of temporal visual attention impossible.

Visual stimuli are normally experienced as long, uninterrupted stretches of experience. In contrast, olfactory experience is temporally discontinuous. It consists of short, discreet, temporally unstructured, olfactory experiences every 1.6 s (Laing, 1983; Mainland and Sobel, 2006). The reason for the interruptions in olfactory experiences is that the access of odorant molecules to the sensory neurons in the nose is controlled by sniffs. Sniffing is a complex behavior and the odor-induced brain responses are coupled to the rhythm of the sniffs (Kepecs et al., 2006). Modulating the sniffing behavior dramatically changes olfactory experiences (Verhagen et al., 2007). It is easy to temporally interrupt an unpleasant olfactory experience by not inhaling and in normal sniffing this interruption occurs every $1.6 \mathrm{~s}$. As a consequence, changes over time that occur in less than a second are not part of olfactory experiences.

Many studies of temporal visual attention involve rapid (several per second) serial visual presentations. The most prominent phenomenon of visual temporal attention is the attentional blink. The attentional blink is the phenomenon that salient visual stimuli are often not consciously detected when they are presented (at the same spatial location) between 200 and $500 \mathrm{~ms}$ after another stimulus (Raymond et al., 1992). In olfaction there is no analog to the attentional blink. The olfactory experience during a sniff is temporally unstructured. The experience in one sniff can be influenced by the previous sniff, but these influences are generally not considered to be an olfactory version of the attentional blink but rather effects of habituation or adaptation.

In summary, attention toward smells can be temporally structured. One can allocate attention toward smells during lunchtime but not for the rest of the day. However, olfaction is a "slower" sense than vision. Photons are faster than the odor molecules diffusing through the olfactory mucus that covers the olfactory sensory neurons in the nose. As a consequence many phenomena of fast temporal attention shifts that are studied in vision have no equivalent in olfaction.

\section{ATTENTION TO OLFACTORY OBJECTS}

Several different notions of "olfactory objects" have been introduced but none of them has become widely accepted. Olfactory objects could be clouds of molecules (Lycan, 2000). Alternatively, it has been suggested that "olfactory sources (objects that produce odors, such as a lion) and olfactory events (odors that emanate from objects, such as a musky lion smell) can be thought of as olfactory objects" (Gottfried, 2010). A third notion has been introduced by Yeshurun and Sobel (2010) who propose that the odor object is the pleasantness of an odor.

The relevant question for the possibility of attention to olfactory objects is if more than a single odor object can be experienced at the same time. If every odor experience consists of a single object, there is nothing for olfactory object-based attention to operate over. The different notions of "olfactory object" lead to different answers to this question. An analogy to vision, where visual objects cannot occupy the same location in visual space at the same time, would suggest that because there is only one location in olfactory space, only one olfactory object can be experienced at any given time. Nevertheless, some researches argue that there can be several olfactory objects simultaneously and that an olfactory object can be separated from an olfactory background (Stevenson and Wilson, 2007).

Because there is no accepted notion of "olfactory object" the disagreement over the possibility of simultaneous olfactory objects and therefore the existence of potential targets for object-based olfactory attention is merely a verbal dispute. I will adopt a notion of "olfactory object" in analogy to the notion of "visual object" according to which an olfactory experience consists of a single olfactory object at any time. There can therefore be no object-based olfactory attention. At any time during an olfactory experience there is only a single olfactory object present. There are no simultaneous objects between which attention could be shifted.

\section{ATTENTION TO FEATURES OF OLFACTORY OBJECTS}

In vision research, the term "feature-based attention" is used to describe two similar types of attention (Carrasco, 2011). The first type of attention is when attention is allocated toward one feature of a visual object, for example color, shape, or orientation, at the expense of other features (Liu, 2003). A second way in which "feature-based attention" is used is to describe attention toward part of the quality space of one feature (Muller, 2006). This is the attentional strategy used in guided search; for example when an object of known color is searched for among other objects. If 
an orange book is searched for on a book shelf, then attention is shifted toward the area of the color quality space surrounding orange and away from all the blue, black, and green books.

Olfactory objects only have a single feature, their smell. There are therefore no features between which olfactory attention could be shifted. Attending to the olfactory modality is attending to the single feature of the single olfactory object that is present at that time. The first type of feature-based attention, attention toward one but not the other features of an object, is therefore not possible in olfaction. However, attention can be selectively allocated to certain smell qualities just as it can be allocated toward certain color qualities. When smelling the complex odor of a glass of wine we seem to be able to attend either to the fruity qualities of the smell or to the woody qualities.

In this type of feature-based attention, attention is shifted within a perceptual quality space. The color quality space is relatively simple. Psychophysical investigation of color vision has shown that mental color qualities can be arranged in a three dimensional space (for example the Munsell color solid) in which the dimensions represent hue, saturation, and lightness (Hardin, 1988). Unfortunately, the situation is much more complex for the odor quality space. Our current understanding of odors and their perception does not allow us to order smells in a quality space similar to the color space. It is possible that the odor space has a complex structure and that multidimensional scaling techniques will eventually discover an ordered multidimensional odor space (Clark, 1993; Lycan, 2000). So far, those attempts have not come close to describing an ordered space. They have, instead, confirmed Plato's view that the most important dimension in odor quality space is pleasantness (Khan et al., 2007). From the fact that no odor quality space has been discovered yet, it can be concluded that if it exists it has a very large number of dimension and a complex structure.

Regardless of its exact structure there is empirical evidence that olfactory attention can be selectively distributed over the odor quality space. The reaction time to an odor for example has been shown to be influenced by expectation. When subjects are looking at a picture of oranges, the reaction time to orange odor is shorter than when looking at a picture of fishes (Gottfried and Dolan, 2003). These authors suggest that this and similar findings are best interpreted as subjects directing their olfactory attention toward a particular (orange-like) odor quality.

In contrast, experiments with odor mixtures have been interpreted as showing that features of an odor cannot be attended to. In these experiments subjects were asked to identify the components of odor mixtures. They were either instructed to report if a single component (for example benzaldehyde) is present in the mixture, or to report all the components (up to six out of six different components) that they can identify in the mixture. There was little difference in the ability to identify components of mixtures between the two conditions (Laing and Glemarec, 1992). However, these results are not relevant for a discussion of feature-based attention because the features of an odor over which attention can operate are perceptual features and not types of molecules. Instead of showing the failure of attention toward features in olfaction these results show that the perceptual features of an odor mixture are not a combination of the perceptual features of its components, a well-established fact (Jinks and Laing, 1999). The results from mixture studies are therefore consistent with the notion that attention can be directed to a specific area of the odor quality space in analogy to feature-based visual attention.

\section{ATTENTION TO OLFACTION}

The fifth type of attention in the taxonomy proposed by Chun et al. (2011) is different from the types discussed so far. In the types of attention discussed so far attention is shifted through space, time, or a quality space within a single modality. In attention toward a modality attention is shifted between modalities. Of these intermodal attentional shifts the shift between audition and vision has been studied in detail (Spence and Driver, 1997). Because of the potential applications in the management of chronic pain, the shifts of attention to and from nociceptive stimuli are also well understood (Eccleston, 1995).

Several psychophysical studies have shown that attention can be shifted toward olfaction in the same way it is shifted toward the other modalities. It has been shown that attention to odors decreases the response time to odors (Spence et al., 2000, 2001a,b). In one study, Spence et al. (2001b) instructed subjects to discriminate either between two different intensities of an odor or between two different light intensities emitted by a diode. The odor was presented through a tube in the subject's nose. There was a continuous flow of odorless air through the tube and when an odor had to be presented, a computerized valve opened, thereby mixing the odorless air with the odor. An auditory cue primed the subjects to attend to either the light (low-pitched tone) or the odor (highpitched tone). The cue was valid in most - but not all - cases. Subjects had a shorter response time to the odor when the cue was valid because they were attending to olfaction rather than to vision.

In addition to these behavioral effects of attending to olfactory information physiological effects of attending to smells have been described. Olfactory event-related brain potentials show attentional modulation (Pause et al., 1997; Krauel et al., 1998; Geisler and Murphy, 2000; Masago et al., 2001) and more recently attention to odors has been shown to alter both behavioral responses to odors and patterns of brain activity in response to odors in functional magnetic resonance imaging (fMRI) experiments (Sabri et al., 2005; Zelano et al., 2005; Plailly et al., 2008). Together these data show that it is possible to attend to olfaction in much the same way in which we may attend to vision or audition.

\section{TYPES OF ATTENTION FOUND IN OLFACTION}

In this section I have developed a taxonomy of olfactory attention that is modeled on a recent general taxonomy of attention (Chun et al., 2011). I have used types of visual attention as reference points. This direct comparison shows that many types of visual attention are modality-specific adaptations to modality-specific information processing requirements. Each modality is adapted to provide us with specific information about our environment. Some systems are specialized for processing spatial information (like vision and passive touch) and in them a special mechanism for spatial attention has evolved. Temporal attention and featurebased attention are the two types of intramodal attentional shift that are found in both vision and olfaction. Temporal attention 
is probably a universal type of attention. As long as attention can be allocated, it can be allocated in time. Feature-based attention is also found both in vision and in olfaction. However, this does not mean that feature-based attention is not modality-specific. It seems that for example in proprioception there are no features that can be attended to. Like the other types of intramodal attention, feature-based attention is an adaptation to specific functions of some sense organs.

It is often overlooked that modality-specific attentional mechanisms are adaptations to sense organs and not adaptations to stimulus types. Facts about the temporal and spatial structure of human olfactory experiences are contingent on how we evolved to sense odors. Insects smell with their antennae which are continuously exposed to the ever-changing odorous ambient air. If we would have evolved olfactory sensors on the tip of our nose, then our olfactory experiences would be uninterrupted by sniffing and smelling would be much more like hearing. Similarly, some single-celled organisms are entirely covered with chemoreceptors. If we would be covered in odorant receptors over our entire body surface, smelling would be much more like passive touch and we would have evolved the mechanisms for covert spatial olfactory attention. Without turning our head we could shift attention from what we are smelling on our knee to what we are smelling on our neck. The attentional mechanisms in the different modalities have co-evolved with the sensory structures themselves and are independent of the physical nature of the stimuli.

In contrast to intramodal attentional shifts, intermodal shifts of attention like the shift of attention toward the olfactory modality are not adaptations to a certain way of information gathering and processing. These shifts are attentional shifts between information that is processed in different ways in different areas of the brain. They are similar to shifts from external to internal attention and to shifts within internal attention, for example from memories to task rules. I will in the next section introduce the shift of attention from other modalities (or internal attention) toward the olfactory modality as a system for the study of the relation between attention and consciousness. Because this type of attention is found in all modalities (and in internal attention), my hope is that results from this system will generalize over a wide range of systems in which attention and consciousness interact.

\section{NEURAL CORRELATES OF OLFACTORY ATTENTION AND CONSCIOUSNESS}

The anatomical structure underlying the processing of olfactory information is simple and radically different from the one found in the other sensory modalities (Figure 1). It has been suggested that the olfactory system represents the "minimal neuroanatomy for a conscious brain" (Morsella et al., 2010). The simplicity of this system may help reveal the mechanisms underlying attentional selection and conscious processing.

Odors are sensed by olfactory sensory neurons in the nose. These neurons project to a layered structure in the forebrain called "olfactory bulb" (Shepherd et al., 2004). The main connection from the olfactory bulb to higher brain centers is to the piriform cortex (which is sometimes also called the "olfactory cortex" or the "primary olfactory cortex"; Bower, 1991). Unlike the visual cortex, auditory cortex, or somatosensory cortex, the piriform cortex is

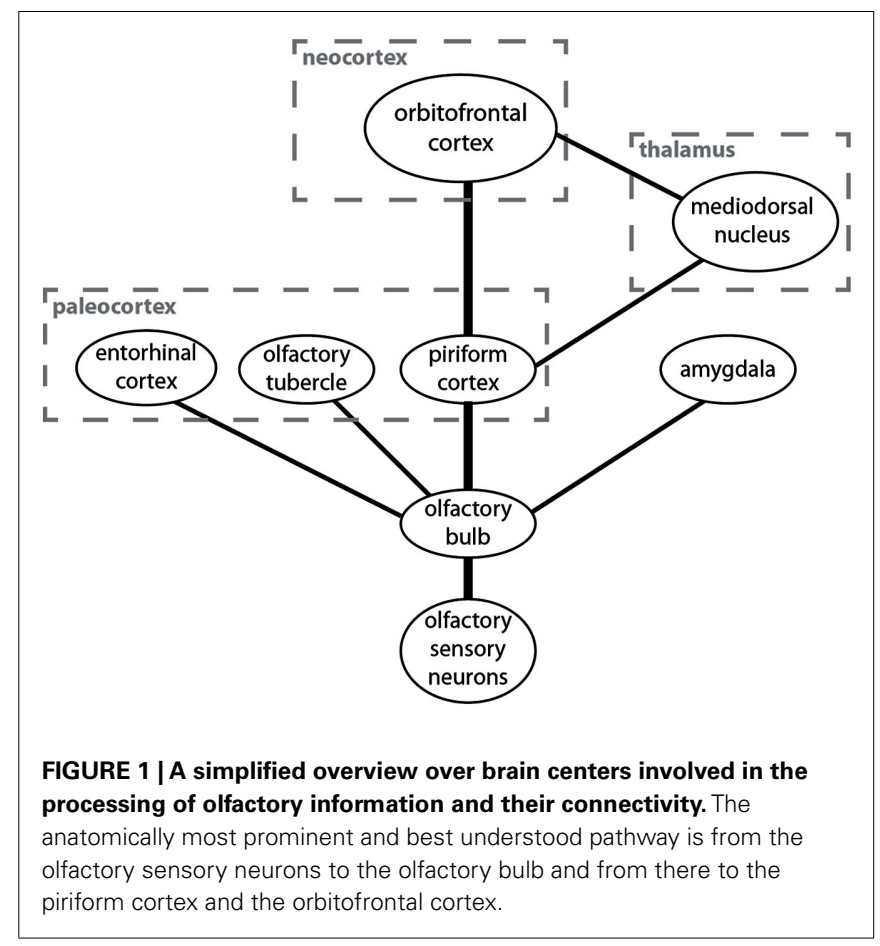

not a neocortical but a paleocortical structure. The paleocortex is phylogenetically older than the neocortex (hence the names) and it consists of three rather than six layers but the cellular architecture is similar. The piriform cortex is the main target for neurons from the olfactory bulb but it is not their only target. They also project to other paleocortical structures like the olfactory tubercle and the entorhinal cortex as well as to the amygdala. I will here only discuss the piriform cortex.

From the piriform cortex the main pathway is directly to the orbitofrontal cortex, which is the neocortical structure in which odor information is processed (Zald and Rauch, 2008). There is also a much less prominent indirect pathway from the piriform cortex to the orbitofrontal cortex via the mediodorsal thalamic nucleus. There are also projections to other brain regions from the piriform cortex, but they are not important for the discussion of neural correlates of attention and consciousness in olfaction.

There are two prominent anatomical differences between the olfactory system and the other sensory systems in the human brain. First, unlike other sensory information the majority of olfactory information never passes through the thalamus. Second, olfactory information is first processed in the paleocortex (specifically in the piriform cortex) and then in the neocortex (specifically in the orbitofrontal cortex). Sensory information from most other modalities is processed only in the neocortex. Two questions arise from these anatomical peculiarities of the olfactory system. (1) What is the functional olfactory analog of the thalamus which is responsible for attentional selection in the other modalities? (2) Is the piriform cortex or the orbitofrontal cortex the functional olfactory analog of the sensory cortices that are responsible for conscious information processing in the other modalities? I will now discuss the two questions and then present a model of attention and consciousness in olfaction based on the answers. 


\section{ATTENTIONAL SELECTION IN OLFACTION}

The attentional selection in visual spatial attention is believed to occur in the thalamus (Crick, 1984; Mcalonan et al., 2008; Geraint, 2009). Furthermore, shifts of attention between modalities are also believed to require the thalamus (Newman, 1995; Smythies, 1997) through thalamic sensory gating (Mccormick and Bal, 1994). However, the majority of olfactory information bypasses the thalamus on its way to the neocortex. This poses the question of which brain structure performs the function of the thalamus in the olfactory system. It has been suggested that attentional selection in olfaction occurs in the olfactory bulb or in the piriform cortex. A third proposal is that the minor indirect pathway that connects the nose to the neocortex via the thalamus is involved in olfactory attention. I will review the evidence for these three proposals.

\section{Attentional selection in the olfactory bulb}

The olfactory bulb is an outgrowth of the forebrain in which the axons of the olfactory sensory neurons terminate (Shepherd et al., 2004). At this first level of processing of olfactory information "molecular features" of odor molecules are encoded, a function that is reminiscent of the encoding of simple features of visual objects in the primary visual cortex (Haberly, 2001). It has also been pointed out that lateral inhibition is found both in the olfactory bulb and in the retina and that these two structures may play similar roles in vision and olfaction, respectively (Devriesa and Baylora, 1993; Nakanishi, 1995; Ghatpande, 2008). Recently it has been proposed that both of these comparisons are valuable and that "the bulb is directly comparable not only to the retina but also to primary visual cortex" (Cleland, 2010).

The motivation to suggest that the olfactory bulb in addition performs the function of the thalamus comes from electrophysiological recordings from neurons in the olfactory bulb of behaving rats. It has been shown that when rats attend to olfaction, neurons in the olfactory bulb fire in tonic mode (as opposed to bursts of spikes when olfaction is not attended to; Kay and Laurent, 1999). This shift between burst and tonic mode depending on attentional state is reminiscent of a similar shift in the thalamus that is associated with attentional processes (Sherman, 2001). There are also broad structural similarities between the thalamus and the olfactory bulb. Most notably, there are excitatory feedback neurons which can produce oscillating patterns of neuronal activity in both systems (Kay and Sherman, 2007).

\section{Attentional selection in the piriform cortex}

From the olfactory bulb, neurons project to the piriform cortex. In analogy to the visual system, it has been suggested that in the piriform cortex the "molecular features" that are processed in the olfactory bulb are combined to form "olfactory objects" (Bower, 1991). The piriform cortex is also the last processing stage for most olfactory information before the neocortex. In all other sensory systems the last processing stage before the neocortex is the thalamus and because of this analogy it has been long speculated that the piriform cortex is an "olfactory thalamus" (Lorente De No, 1938). Support for this model comes from electrophysiological recordings in rats that showed that there are statedependent changes in odorant-evoked responses in a majority of piriform cortex neurons, but not in neurons in the olfactory bulb (Murakami et al., 2005). The apparent discrepancy between this study and the study by Kay and Laurent (1999) may be due to the fact that in this study the states on which the neuronal changes depended were defined by neocortical electroencephalograms (in anesthetized animals), whereas in the study by Kay and Laurent (1999) they were defined behaviorally (in behaving animals).

Two fMRI studies of subjects attending to odors have uncovered attentional modulation in the piriform cortex (Zelano et al., 2005; Veldhuizen and Small, 2011). Two other studies did not show attentional modulation of piriform cortex activity (Sabri et al., 2005; Plailly et al., 2008). The four studies used different methodology, stimuli, and thresholds for statistical significance and the different results may reflect these differences. The effects of piriform cortex lesions can be studied in epilepsy patients who underwent temporal lobectomy (for references, see Gottfried, 2010). These procedures are performed unilaterally. In one study in which patients' olfactory performance was tested before and after the procedure, left-resected patients did show no effect of the procedure on olfactory perception whereas right-resected patients showed some decline. The ability to attend to the olfactory modality has to my knowledge not been tested in subjects with piriform cortex lesions.

\section{Attentional selection in the thalamus}

The piriform cortex is for most olfactory information the last processing stage before the neocortex. However, in addition to the main direct pathway between piriform cortex and neocortex, there is also a minor indirect pathway via the mediodorsal thalamus (Carmichael et al., 1994). This indirect pathway has been demonstrated anatomically and electrophysiologically in rats (Price and Slotnick, 1983; Price, 1985), rabbits (Benjamin et al., 1978), and monkeys (Yarita et al., 1980; Takagia, 1986). At least in rats it is very sparse (Price, 1985; Price et al., 1991). The existence of this indirect pathway suggests the attractive possibility that attentional selection of all sensory information including olfactory information occurs in the thalamus.

Consistent with this idea it was shown in an elegant study that attention to the olfactory modality strengthened the connectivity between the mediodorsal thalamic nucleus and the neocortex (specifically the orbitofrontal cortex), suggesting that a thalamic relay for olfactory information is activated by attending to olfaction (Plailly et al., 2008). Traditional fMRI studies do not measure the connectivity between different brain areas but merely the response magnitude in different parts of the brain. Out of three traditional fMRI studies of attention to olfaction only one identified the thalamus as a structure that is involved in attention toward olfaction (Veldhuizen and Small, 2011). Instead, these studies found attentional modulation in the orbitofrontal cortex (Sabri et al., 2005; Veldhuizen and Small, 2011), the olfactory tubercle (Zelano et al., 2005), the cerebellum (Zelano et al., 2005; Veldhuizen and Small, 2011) as well as in several other structures (Veldhuizen and Small, 2011).

The results of studies of olfactory defects in patients with lesions in the mediodorsal thalamus (Potter and Butters, 1980; Rousseaux et al., 1996; Asai et al., 2008; Sela et al., 2009; Tham et al., 2011a,b) have been interpreted as showing that it is "likely" that patients 
with mediodorsal thalamic nucleus lesions have an impairment in olfactory attention (Tham et al., 2011a). However, the findings in these patients are not consistent with the idea that in the absence of the indirect pathway from the piriform cortex to the orbitofrontal cortex via the mediodorsal thalamus the olfactory modality cannot be attended to.

A further problem for any model that assigns the mediodorsal thalamus a role in attending to olfaction is that the indirect connection between the piriform cortex and the orbitofrontal cortex via the thalamus has not been found (to my knowledge) in humans yet. Even if large scale neuroanatomy projects like the Human Connectome Project (Wig et al., 2011) will show that this connection exists in humans, it is unclear how much information can be transferred through such a sparse connection. Discussing the indirect pathway in the rat brain, Price et al. (1991) remarked that it is "not well equipped to convey detailed sensory information to the thalamus for relay to the neocortex."

In summary, it is not plausible that the indirect thalamic pathway is responsible for shifts of attention toward the olfactory modality. The weak effects of thalamic lesions suggest a more subtle role of the thalamus in olfaction. If not in the thalamus, attentional selection has been speculated to occur in the olfactory bulb or the piriform cortex. The evidence for attentional selection in the piriform cortex is more convincing although a study of olfactory attention in patients with lesions of the piriform cortex would help strengthen the evidence considerably.

The thalamus plays a role both in intramodal attentional shifts in vision (Crick, 1984; Mcalonan et al., 2008; Geraint, 2009) and in intermodal shifts for example between vision and audition (Newman, 1995; Smythies, 1997). According to the view presented here, in olfaction the piriform cortex plays the role of the thalamus in intramodal attentional shifts between, for example, fruity and flowery odors. Furthermore, the piriform cortex is also necessary for shifts to and from olfaction. Accordingly, the role of the thalamus in attentional shifts is not universal but modalityspecific. Attention can be shifted from memories to task rules, from thoughts to olfaction, from vision to audition, or from olfaction to vision. Different brain structures are involved in these different types of attentional shifts. There has to be a connection between the different structures to coordinate their activity. Maybe the few fibers that connect the piriform cortex with the thalamus (Carmichael et al., 1994) do not relay information about the olfactory stimulus to the thalamus but are involved in the coordination of attentional shifts between olfaction and the other sensory modalities.

\section{NEURAL CORRELATES OF CONSCIOUS OLFACTORY PROCESSING}

According to computational theories of consciousness being conscious is a property of certain types of information processing (Cleeremans, 2005). Structures in the brain in which information is processed consciously have been called the neural correlates of consciousness (Metzinger, 2000; Tononi and Koch, 2008). Somewhere in the brain olfactory information is processed consciously and the structure in which this computation happens is the neural correlate of olfactory consciousness. Since there are several concepts of consciousness, there can be several neural correlates of olfactory consciousness. I will discuss here the phenomenal neural correlate of consciousness (Block, 2005; Stevenson, 2009) which is the structure in the brain in which information about the content of olfactory experience is processed consciously.

Olfactory information is processed both in the paleocortical piriform cortex and in the neocortical orbitofrontal cortex. The contribution of these two cortical sites to conscious processing of olfactory information is unclear (Shepherd, 2007). The paleocortical piriform cortex is a three-layered cortex that is only a single synapse away from the odor molecules. It is structurally simpler and phylogenetically older than the six-layered neocortical orbitofrontal cortex which is only found in mammals (Gottfried, 2007).

There are reasons to assume that the phenomenal neural correlate of olfactory consciousness is found in the neocortical orbitofrontal cortex. First, the neural correlates of visual consciousness, which have been explored in some detail, are also assumed to be found in neocortical structures and associated brain regions (Crick and Koch, 2003). Second, a recent lesion study of a single patient showed that brain injury that was largely limited to the right orbitofrontal cortex did completely abolish conscious processing of olfactory information. In contrast, the patient's ability to modulate his sniffing behavior in response to olfactory stimuli was unaffected and he showed normal skin conductance responses to odors ( $\mathrm{Li}$ et al., 2010). This study is consistent with previous studies of patients with orbitofrontal damage or lesions (see references in Li et al., 2010).

However, the most important question for locating the neural correlate of phenomenally conscious olfactory experiences is what the content of those experiences is and where the information specifying these contents is processed. I will now first describe the phenomenal content of conscious olfactory experiences and then review what type of information is processed in the piriform cortex and in the orbitofrontal cortex. A comparison will show that the neural correlate of olfactory consciousness is likely to be found somewhere in the orbitofrontal cortex.

\section{The content of olfactory consciousness}

The content of olfactory consciousness is determined partially by the physical features of the odor molecule. There are prominent physical features that have a strong influence on the content of olfactory consciousness. For example sulfur atoms in an odor molecule result reliably in a characteristic sulfuric smell (Boelens and van Gemert, 1993). However, the content of olfactory consciousness is also strongly influenced by contextual factors. The influence of contextual factors on the content of olfactory consciousness means that, even in theory, complete knowledge of the physical features of the odor molecule is not sufficient to accurately predict the content of the conscious olfactory experience. I use "contextual factors" here as an umbrella term for remembered associations with the odor, internal states (hunger, sadness, etc.), and sensory input from other modalities. I will now discuss the influence of each of these types of context.

Associations with the odor that were established during a previous encounter can change how the odor is experienced. Tequila smells different before and after tequila-induced alcohol poisoning. In the laboratory, changes like this have been demonstrated in a series of experiments that investigated how odors can acquire 
the odor quality "sweet" through association. Several experiments (reviewed in Stevenson and Boakes, 2003) have shown that when odors are repeatedly paired with sugar, they acquire the odor quality "sweet" and are subsequently described as sweet smelling when they are experienced in the absence of sugar.

The influence of internal states on the content of olfactory consciousness is seen in the satiety dependent difference in how the same odor stimulus is perceived at different times. It has been suggested that the smell of a "grilled 40-oz. dry-aged porterhouse steak" is experienced differently after one has just consumed such a steak than before (Gottfried, 2007). Similarly, Yeshurun and Sobel (2010) write that "banana odor when you are hungry is a different object from banana odor when you are satiated."

The most dramatic example of information from other sensory modalities that modulates olfactory experiences is the perception of flavor. Flavor is a combination of olfactory, gustatory, tactile, and other sensations. The olfactory component in flavor is not experienced as a distinct experience but as part of a flavor experience.

These examples demonstrate the strong contribution of contextual factors to the content of olfactory conscious experience. This is not a novel proposal. Others have defended similar positions in much more detail. Gottfried and Wu (2009) called this effect the "perceptual pliability of odor objects," whereas Yeshurun and Sobel (2010) called odor a "sensory emotion." Stevenson and Boakes (2003), who focused on the effect of memories and association on odor experiences, proposed "a mnemonic theory of odor perception."

\section{What information is processed in the piriform cortex and in the orbitofrontal cortex}

If both the physical features of the odor molecules and the context contribute to the content of conscious olfactory experience then the neural correlate of consciousness must be located in a structure that processes both information about the stimulus and information about the context. The two candidate structures for the neural correlate of consciousness are the piriform cortex and the orbitofrontal cortex and I will now discuss to what extent information about context is processed in these two structures.

Information processing in the piriform cortex (Bower, 1991; Neville and Haberly, 2004) and the orbitofrontal cortex (Gottfried and Zald, 2005; Zald and Rauch, 2008) has been comprehensively reviewed elsewhere. I will focus here on studies in which olfactory stimuli are presented in different contexts to identify brain structures in which activity is influenced both by the stimulus and by the context. fMRI studies allow to directly compare activity in the piriform cortex and the orbitofrontal cortex under these conditions. Several such studies have been performed and the results show clearly that context is processed mainly or exclusively in the orbitofrontal cortex. Remembered associations with the odor, internal states, and sensory input from other modalities have all been investigated.

Changes of perceived odor quality through remembered associations have been studied in most detail psychophysically for the acquisition of a "sweet" odor quality by odors that are paired with sugars (reviewed in Stevenson and Boakes, 2003). To my knowledge it has not been investigated in which brain regions the representation of odors changes when they acquire the "sweet" odor quality. However, it has been shown that orbitofrontal cortex activity depends on how likely it is that a given odortaste combination has been experienced previously. There is a superadditive response in the orbitofrontal cortex to pairs that have presumably been experienced together before (vanillasugar), but not to pairs that presumably have not been experienced before (vanilla-salt; Small et al., 2004). Another study has investigated the processes of pairing an odor with an electric shock (Li et al., 2008). Pairing an odor with an electric shock changes the odor representation in both the piriform and the orbitofrontal cortex. However, the changes in the piriform cortex are qualitative whereas the changes in the orbitofrontal cortex are quantitative.

Among internal states, the influence of satiety on olfactory information processing has been studied extensively. It has been shown that odor representation in the orbitofrontal cortex (but not in the piriform cortex) is satiety dependent (O'Doherty et al., 2000). The odor of banana in these experiments has been shown to be represented differently in the orbitofrontal cortex after subjects ate banana to satiety. The representation of the odor of unrelated food did not change.

Odor representations in the orbitofrontal cortex also change when odors are paired with stimuli in other modalities. Neural activation in the orbitofrontal cortex was increased, for example, when an odor was presented in the expected visual context, for example when a rose odor was combined with the picture of a rose compared to the same odor combined with a picture of a bus (Gottfried and Dolan, 2003). This effect is not limited to pictures but extends to visually perceived verbal labels (De Araujo et al., 2005). The same odor (isovaleric acid) elicited different orbitofrontal cortex responses when labeled "cheddar cheese" than when labeled "body odor." In this study the results for the piriform cortex were ambiguous and the authors state that there "may" be modulation of the piriform cortex response. In addition to visual stimuli, gustatory stimuli can also influence the odor representation in the orbitofrontal cortex (De Araujo et al., 2003).

Taken together these results show that much of the information about context that contributes to the content of olfactory consciousness is processed in the orbitofrontal cortex but not in the piriform cortex. Although it is clear from the results of some of the studies in humans that were discussed above as well as from electrophysiological recordings in rodents (Wilson, 2010; Doucette et al., 2011) that some types of context can influence processing in the piriform cortex, the most prominent function of the piriform cortex is to process information about physical features of odor molecules. In contrast, the most prominent function of the orbitofrontal cortex is to integrate information about physical features of the stimulus with information about the context. The information processed in the orbitofrontal cortex is the information that determines the content of conscious olfactory experiences.

\section{NEURAL CORRELATES OF OLFACTORY ATTENTION AND CONSCIOUSNESS}

In this section I introduced three candidate structures for attentional selection and two candidate structures for conscious 


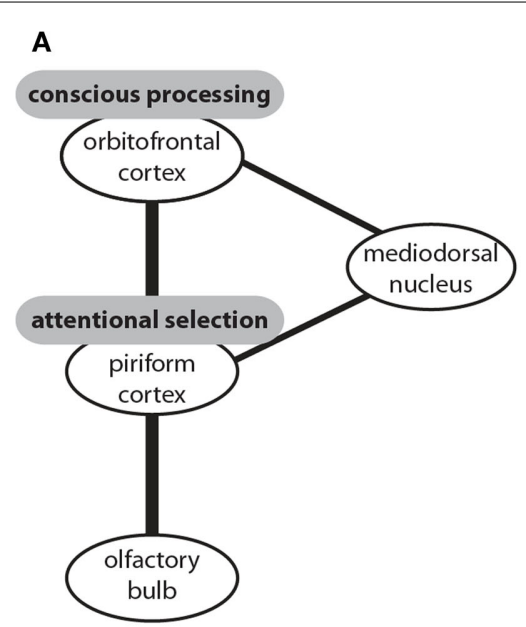

D

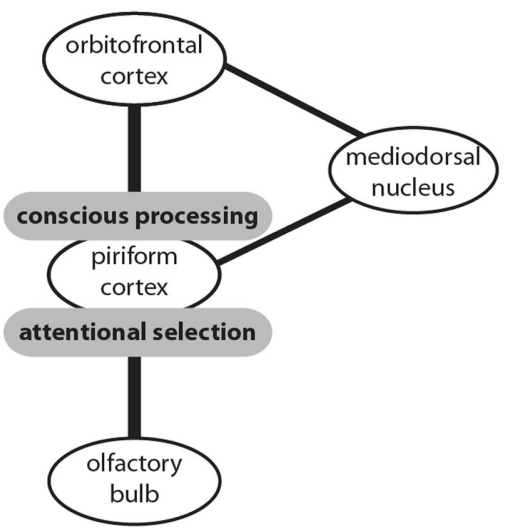

B

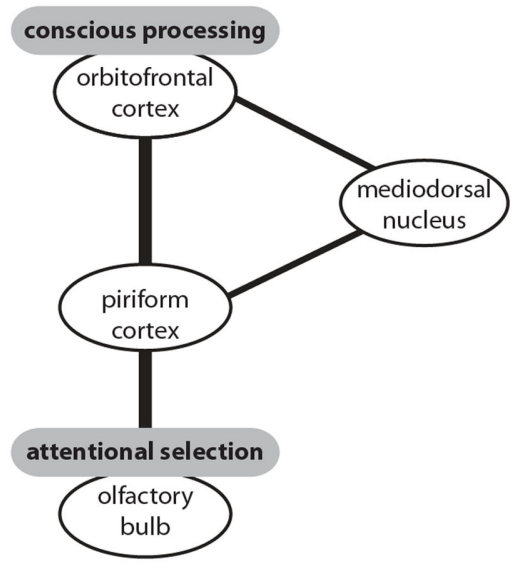

E

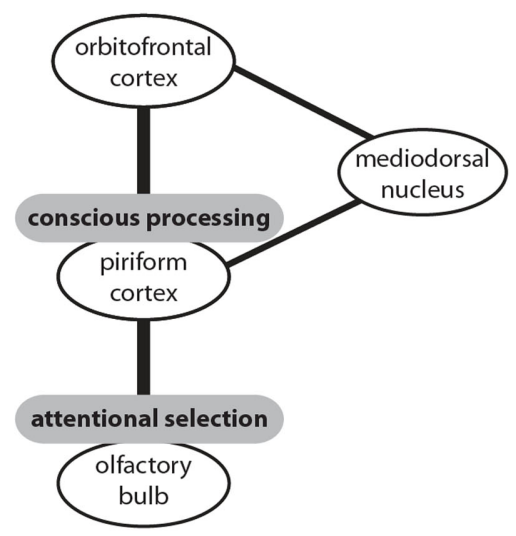

C

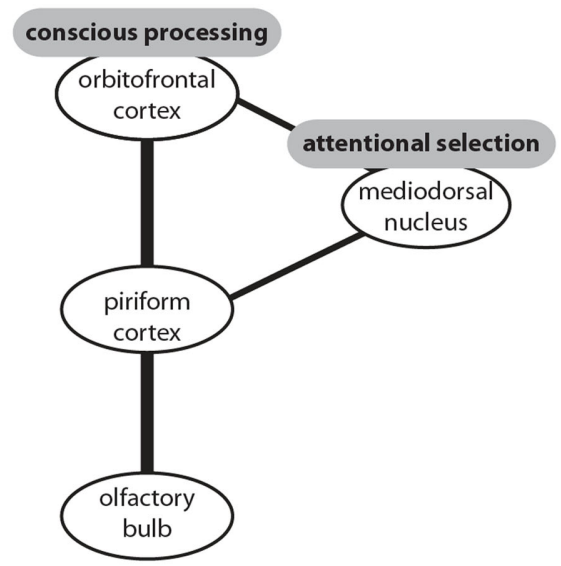

$\mathbf{F}$

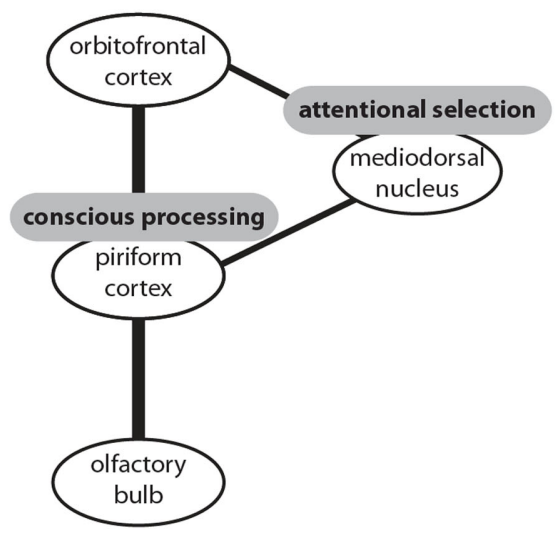

FIGURE 2 |The connectivity of brain centers involved in the processing of olfactory information and sites that have been proposed to be the neural correlates of attentional selection and conscious processing.

Three potential sites of attentional selection and two potential sites of conscious processing can be combined to six models (A-F). Conscious processing of olfactory information could occur in the orbitofrontal cortex with attentional selection occurring prior to conscious processing either in the piriform cortex $(\mathbf{A})$, or the olfactory bulb $(\mathbf{B})$, or the mediodorsal nucleus of the thalamus (C). Alternatively, conscious processing could occur in the piriform cortex with attentional selection occurring in the same structure (D), or prior to conscious processing in the olfactory bulb (E), or after conscious processing in the mediodorsal nucleus of the thalamus. processing in the olfactory system. These structures could be combined to six models of olfactory information processing (Figure 2). In four of these models attentional selection occurs prior to conscious processing. In one model attentional selection operates over consciously processed information (Figure $2 \mathbf{F}$ ) and in another model both attention and conscious processing are accomplished by the piriform cortex (Figure 2D). To my knowledge, only three of these models have been suggested. It has been proposed that that attentional selection occurs in the olfactory bulb and conscious processing in the piriform cortex (Figure 2E; Kay and Sherman, 2007). Alternatively, it has been suggested that, like in other sensory modalities, attentional selection occurs in the thalamus and conscious processing in the neocortical orbitofrontal cortex (Figure 2C; Plailly et al., 2008). The data I reviewed here supports the third model which proposes that the piriform cortex performs attentional selection and the orbitofrontal cortex conscious processing of olfactory information (Murakami et al., 2005; Figure 2A).

\section{IS ATTENTION TOWARD THE OLFACTORY MODALITY NECESSARY FOR OLFACTORY CONSCIOUSNESS?}

Identifying the neural structures underlying attention and consciousness is an important step for the conceptual analysis of the relation between the two processes. Many questions can be and have been asked about the relation of attention to consciousness. Is attention the same as consciousness (Posner, 1994; Lamme, 2003)? Is attention necessary and/or sufficient for consciousness (Prinz, 2010; Van Boxtel et al., 2010)? Or is it the other way around and consciousness is necessary and/or sufficient for attention (Mole, 2008)? Is information first consciously processed and then attention acts on it or does attention act on information before it is consciously processed (Lamme, 2003)? All these are fundamental conceptual questions that remain unanswered. I will focus here on the question about the necessity of attention for consciousness.

The question if attention is necessary for consciousness has been investigated previously in some types of visual attention using a variety of paradigms. Inattentional blindness (Mack and Rock, 
1998), attentional blink (Raymond et al., 1992), change blindness (Simons and Rensink, 2005), and load induced blindness (Macdonald and Lavie, 2008) are striking phenomena in visual perception in which stimuli that are consciously processed when attended to are not consciously processed when not attended to. On the other hand, there are other phenomena in which stimuli are consciously processed in the absence of attention (see Van Boxtel et al., 2010 and references therein). A (possibly insurmountable) weakness of these experiments is that it is currently not possible to achieve and demonstrate complete absence of all attention to an area of the visual field (De Brigard and Prinz, 2010).

I will now attempt to answer the question about the necessity of attention for consciousness for the olfactory modality. I will present two types of evidence, observational and experimental, that attention is necessary for conscious processing of olfactory information and then address the main objection to this claim. Based on the evidence, I will argue that attention to smells is necessary for consciousness of smells.

\section{OBSERVATIONAL EVIDENCE THAT ATTENTION IS NECESSARY FOR OLFACTORY CONSCIOUSNESS}

Olfaction researchers often seem to presuppose that attention is necessary for olfactory consciousness. Sela and Sobel (2010) for example write that "olfactory stimuli are less prone to attract attention, and therefore humans have poor awareness to the olfactory environment." Similarly, Plailly et al. (2008) conclude from their finding that the thalamic relay is involved in directing attention to smells that olfaction requires a thalamic relay for the conscious processing of smells, a conclusion that presupposes that directing attention to smells is necessary to consciously process them

The assumption that attention to olfaction is necessary for consciousness of olfaction is consistent with most normal, everyday olfactory experiences. With almost every breath we inhale air containing odors at relatively high concentrations; yet olfactory experiences are very rare (compared, for example, with visual experiences). This shows that there is an additional cognitive factor necessary to turn the presence of an olfactory stimulus into conscious olfactory experience. Because attending to the olfactory modality almost always results in a conscious olfactory experience, it seems plausible that this cognitive factor is attention.

The importance of the role of attention for olfactory experiences is further illustrated by the fact that people are very often wrong in their judgments about changes in their own olfactory abilities or in the odorous environment. The natural assumption, when a person's conscious olfactory experiences change systematically is that either the stimuli or the sensory apparatus has changed. However, in numerous well studied situations this is not the case. Instead, the change in conscious olfactory experience is entirely caused by a change in attention to the olfactory modality. Increased consciousness of smells due to increased attention to smells is seen during pregnancy, but also in people in which the cause for the change is not known.

The vast majority of pregnant women report that their olfactory sensitivity is enhanced during pregnancy. Studies have shown that this is not the case. The olfactory acuity is not changed during pregnancy (Cameron, 2007; Doty and Cameron, 2009). Instead the systematic differences in conscious olfactory experiences are caused by attentional factors. The involuntary increase in attention to odors during pregnancy is probably an adaptive response to the fact that the fetus is especially volatile to environmental poisons and spoiled food. These attentional changes result in a radically altered olfactory conscious experience.

In the case of heightened odor awareness during pregnancy the cause for the attentional shift toward olfaction is known, but there are also vast differences in the attention to olfaction among non-pregnant individuals. Psychophysically measured olfactory abilities, in the general population, do not correlate with how annoyed subjects are by environmental odors or with how the subjects rate their own olfactory abilities. How annoyed they are by odors and how they rate their abilities however does correlate (Knaapila et al., 2008). This shows that people attribute changes in their conscious olfactory experience that are caused by attentional processes to changes in their olfactory abilities. In extreme cases, heightened attention to the olfactory modality can cause debilitating conditions that are part of a heterogeneous group of conditions called "multiple chemical sensitivity" or "idiopathic environmental intolerances" (Dalton and Hummel, 2000).

\section{EXPERIMENTAL EVIDENCE THAT ATTENTION IS NECESSARY FOR OLFACTORY CONSCIOUSNESS}

These observations about the fundamental role of attention in olfactory consciousness suggest that attention is necessary for consciousness in olfaction. Unfortunately, there is no empirical study directly addressing the question of necessity that could support this conclusion. All studies of the role of attention in olfactory consciousness present the stimulus through a plastic tube that is inserted into the subject's nostril. It can be assumed that under this condition subjects will always attend to some degree to the olfactory modality, regardless of the experimenter's instructions. This experimental setup is therefore not suitable to study olfactory consciousness in the absence of attention to olfaction.

Alternative experimental setups make it possible to reduce the subjects' attention to the olfactory modality further. The most covert way to deliver an odor stimulus is to add it to the ambient air that the subject is breathing. Such experiments have revealed a stunning failure of subjects to become conscious of unattended smells (Lorig, 1992; Degel and Koester, 1999). In one study (Lorig, 1992), in which the influence of odors on the appeal of pictures was studied, only 3 out of the 93 subjects became aware of the odor manipulations whereas several other subjects reported a perceived (although non-existent) change in luminance.

Studies like this demonstrate dramatically the absence of olfactory consciousness in the absence of attention. However, what would be needed to test the necessity of attention for odor consciousness would be a study that determines the detection threshold for ambient odor in a situation in which subjects are engaged in some non-olfactory task and have no indication that odors are part of the experiment. This could then be compared to the detection threshold for ambient odor when subjects are told to attend to ambient odors. My prediction is that such an experiment would reveal a very strong influence of attention on olfactory consciousness. However, I also predict that at very high odor concentrations subjects would consciously perceive the odor even under minimal attention conditions. This would be an apparent counterexample to the claim that attention is necessary for olfactory consciousness. 


\section{THE STRONG STIMULUS OBJECTION AGAINST THE NECESSITY OF ATTENTION FOR OLFACTORY CONSCIOUSNESS}

Examples of apparent olfactory consciousness in the absence of attention to olfaction can also be found in every-day experiences. Ethyl mercaptan, which is added as a warning agent to propane gas to alert us to gas leaks, for example, is often perceived by people who do not attend to smells at all. It has to be added at 57,000-times the concentration at which it is detected when it is attended to (Sela and Sobel, 2010), but a case of conscious detection in the absence of attention would refute the claim that attention is necessary regardless of the stimulus intensity. This objection is not limited to the cases of attention in the olfactory system. In the visual system the phenomenon of change blindness seems to show that attention is necessary for conscious perception. However, when the change that is introduced outside the attended area is a large red triangle in a black and white image, then the change will be processed consciously although it occurred in an unattended area.

A usual response to this objection is to distinguish between top-down attention, which is a cognitive process, and bottom-up attention, which is a feature of the stimulus. I prefer to not call anything "attention" that is not a cognitive process and therefore do reject this response. Instead, I have two responses to the strong stimulus objection. The first is that it is possible that attention to olfaction is never completely absent and that the minimum attention that could possibly be allocated to the olfactory modality is sufficient for conscious processing of ethyl mercaptan at 57,000times the concentration at which it is consciously processed when attended to. The second response is that not everything that is attributed to the sense of smell is actually mediated by the sense of smell. There is a second sensory system that senses volatile chemicals in our nose.

Although it has not been demonstrated experimentally, it is plausible that the extent to which attention is allocated to the olfactory modality allows of degrees. "Attended to" and "not attended to" are most likely idealized extreme cases of a continuous process. If this is the case then subjects would perform best at tasks requiring conscious processing of olfactory information when instructed to attend to olfaction. The performance would drop in the resting state and further drop when they are instructed to attend to the visual modality. If they perform a complex visual task for which they are highly motivated, the olfactory performance would drop even further.

If it is true that attention to olfaction allows for degrees then the question is over which range attention to olfaction can be modulated and if it is ever completely absent. A case in which one may expect attention to olfaction to be minimal is sleep. And indeed, odor stimuli at very high concentration, unlike stimuli in other modalities, fail to awake sleeping subjects (Carskadon and Herz, 2004). This is consistent with the notion that attention is necessary for consciousness of smells. The apparent counterexamples are cases in which minimal attention is allocated to olfaction. Because of the intensity of the stimulus and the long time the stimulus is present this minimal attention is sufficient for conscious processing.

The second response to the strong stimulus objection is to point out that experience is not a good guide to judge if there are smells that are consciously processed in the absence of attention to olfaction because what is experienced as a "smell" is a multimodal sensory experience, only partially mediated by the olfactory system. It may be surprising, for example, that smells can't wake sleeping subjects (Carskadon and Herz, 2004). Sleepers often get woken up by the smell of smoke and "smelling salts" have brought countless fainted women in Victorian England and knocked-out boxers back to consciousness. However, neither smoke nor the ammonia gas released by smelling salts is perceived by the olfactory system. Instead, these and many other stimuli are perceived by nerve endings of the trigeminal nerve in the nasal cavity (Doty, 1995). The trigeminal nerve is a warning system that evolved to prevent us from inhaling dangerous substances. As such, it is always attended to and if the stimuli are strong enough to activate it (it is much less sensitive than the olfactory system), the information will be consciously processed. Because both the olfactory system and the trigeminal nerve detect stimuli inside the nasal cavity, the perceptions mediated by them are often collectively referred to as "smelling." However, unlike olfactory information, trigeminal information is not processed in the paleocortex and instead, like all sensory non-olfactory information, is relayed through the thalamus to the neocortex (Huart et al., 2009). Apparent cases of "smells" being perceived without being attended to are likely to be mediated by this warning system and not by the olfactory system.

In summary, the fact that very strong olfactory stimuli are often consciously processed by subjects who are not attending to olfaction seems to show that attention is not necessary for olfactory consciousness. However, two things have to be considered. First, it is unlikely that there are many situations in which subjects do not attend to one of their five senses at all. And second, there is a dedicated warning system to alert us to dangerous volatile chemicals, the trigeminal nerve, which may be responsible for most of the cases in which volatile chemicals are sensed in the absence of attention to olfaction. The strong stimulus objection is therefore no reason to assume that attention is not necessary for consciousness of smells.

\section{CONCLUSION}

My goal in this review was to introduce attention to the olfactory modality as a simple system in which to study the relation between consciousness and attention. Toward this goal I first showed how this system fits into a general taxonomy of attention. I then discussed the neuroanatomy of the olfactory system to illustrate how this system differs from the more complicated visual system. I also demonstrated the utility of this model system by using it to weigh in on the question of the necessity of attention for consciousness.

The visual system, despite being extremely specialized and complicated, has become the de facto model system for perceptual cognitive neuroscience. I hope that I succeeded here in showing the potential of very simple and basic systems like olfaction for revealing basic conceptual truths about entities like "attention" and "consciousness."

\section{ACKNOWLEDGMENTS}

My research was supported by a Branco Weiss Fellowship of the Society in Science Foundation and a NARSAD Young Investigator Award. I am grateful to, among many others, Jessica Keiser, Michael Crickmore, Richard Stevenson, and Jesse Prinz for discussions and helpful comments on earlier versions of this manuscript. 


\section{REFERENCES}

Asai, H., Udaka, F., Hirano, M., and Ueno, S. (2008). Odor abnormalities caused by bilateral thalamic infarction. Clin. Neurol. Neurosurg. 110, 500-501.

Benjamin, R. M., Jackson, J. C., and Golden, G. T. (1978). Cortical projections of the thalamic mediodorsal nucleus in the rabbit. Brain Res. 141, 251-265.

Block, N. (2005). Two neural correlates of consciousness. Trends Cogn. Sci. (Regul. Ed.) 9, 46-52.

Boelens, M. H., and van Gemert, L. J. (1993). Volatile character-impact sulfur compounds and their sensory properties. Perfumer Flavorist $18,29-39$.

Bower, J. M. (1991). "Piriform cortex and olfactory object recognition," in Olfaction: A Model for Computational Neuroscience, eds J. L. Davis and $\mathrm{H}$. Eichenbaum (Cambridge: MIT Press), 265-286.

Cameron, L. E. (2007). Measures of human olfactory perception during pregnancy. Chem. Senses 32, 775-782.

Carmichael, S. T., Clugnet, M. C., and Price, J. L. (1994). Central olfactory connections in the macaque monkey. J. Comp. Neurol. 346, 403-434.

Carrasco, M. (2011). Visual attention: the past 25 years. Vision Res. 51, 1484-1525.

Carskadon, M. A., and Herz, R. S. (2004). Minimal olfactory perception during sleep: why odor alarms will not work for humans. Sleep 27, 402-405.

Chun, M. M., Golomb, J. D., and TurkBrowne, N. B. (2011). A taxonomy of external and internal attention. Annu. Rev. Psychol. 62, 73-101.

Clark, A. (1993). Sensory Qualities. Cambridge: Cambridge University Press.

Cleeremans, A. (2005). Computational correlates of consciousness. Prog. Brain Res. 150, 81-98.

Cleland, T. A. (2010). Early transformations in odor representation. Trends Neurosci. 33, 130-139.

Coull, J. T., and Nobre, A. C. (1998). Where and when to pay attention: the neural systems for directing attention to spatial locations and to time intervals as revealed by both PET and fMRI. J. Neurosci. 18, 7426-7435.

Crick, F. (1984). Function of the thalamic reticular complex - the searchlight hypothesis. Proc. Natl. Acad. Sci. U.S.A. 81, 4586-4590.

Crick, F., and Koch, C. (2003). A framework for consciousness. Nat. Neurosci. 6, 119-126.
Dalton, P., and Hummel, T. (2000). Chemosensory function and response in idiopathic environmental intolerance. Occup. Med. (Chic. Ill.) 15, 539-556.

De Araujo, I. E., Rolls, E. T., Velazco, M. I., Margot, C., and Cayeux, I. (2005). Cognitive modulation of olfactory processing. Neuron 46, 671-679.

De Araujo, I. E. T., Rolls, E. T., Kringelbach, M. L., Mcglone, F., and Phillips, N. (2003). Taste-olfactory convergence, and the representation of the pleasantness of flavour, in the human brain. Eur. J. Neurosci. 18, 2059-2068.

De Brigard, F., and Prinz, J. (2010). Attention and consciousness. Wiley Interdiscip. Rev. Cogn. Sci. 1, 51-59.

Degel, J., and Koester, E. P. (1999). Odors: implicit memory and performance effects. Chem. Senses 24, 317-325.

Dehaene, S., Changeux, J., Naccache, L., Sackur, J., and Sergent, C. (2006). Conscious, preconscious, and subliminal processing: a testable taxonomy. Trends Cogn. Sci. (Regul. Ed.) 10, 204-211.

Devriesa, S. H., and Baylora, D. A. (1993). Synaptic circuitry of the retina and olfactory bulb. Cell 72 , 139-149.

Doty, R. L. (1995). "Intranasal trigeminal chemoreception: anatomy, physiology, and psychophysics," in Handbook of Olfaction and Gustation, ed. R. L. Doty (New York: Marcel Dekker, Inc.), 821-834.

Doty, R. L., and Cameron, E. L. (2009). Sex differences and reproductive hormone influences on human odor perception. Physiol. Behav. 97, 213-228.

Doucette, W., Gire, D. H., Whitesell, J., Carmean, V., Lucero, M. T., and Restrepo, D. (2011). Associative cortex features in the first olfactory brain relay station. Neuron 69, 1176-1187.

Eccleston, C. (1995). The attentional control of pain - methodological and theoretical concerns. Pain 63, 3-10.

Forster, B., and Eimer, M. (2004). The attentional selection of spatial and non-spatial attributes in touch: ERP evidence for parallel and independent processes. Biol. Psychol. 66, $1-20$.

Frasnelli, J., Charbonneau, G., Collignon, O., and Lepore, F. (2008). Odor localization and sniffing. Chem. Senses 34, 139-144.
Geisler, M. W., and Murphy, C. (2000). Event-related brain potentials to attended and ignored olfactory and trigeminal stimuli. Int. J. Psychophysiol. 37, 309-315.

Geraint, R. (2009). Visual attention: the thalamus at the centre? Curr. Biol. 19 , R213-R214.

Ghatpande, A. S. (2008). Is the olfactory bulb computationally similar to the retina? J. Neurophysiol. 101, 1-4.

Gottfried, J. A. (2007). What can an orbitofrontal cortex-endowed animal do with smells? Ann. N. Y. Acad. Sci. 1121, 102-120.

Gottfried, J. A. (2010). Central mechanisms of odour object perception. Nat. Rev. Neurosci. 11, 628-641.

Gottfried, J. A., and Dolan, R. J. (2003). The nose smells what the eye sees: crossmodal visual facilitation of human olfactory perception. Neuron 39, 375-386.

Gottfried, J. A., and Wu, K. N. (2009). Perceptual and neural pliability of odor objects. Ann. N. Y. Acad. Sci. 1170, 324-332.

Gottfried, J. A., and Zald, D. H. (2005). On the scent of human olfactory orbitofrontal cortex: metaanalysis and comparison to nonhuman primates. Brain Res. Rev. 50 287-304.

Haberly, L. B. (2001). Parallel distributed processing in olfactory cortex: new insights from morphological and physiological analysis of neuronal circuitry. Chem. Senses 26, 551-576.

Hardin, C. L. (1988). Color for Philosophers. Indianapolis: Hackett Publishing Company.

Huart, C., Collet, S., and Rombaux, P. (2009). Chemosensory pathways from periphery to cortex. B-ENT 5(Suppl. 13), 3-9.

Isaacson, J. S. (2010). Odor representations in mammalian cortical circuits. Curr. Opin. Neurobiol. 20, 328-331.

Jinks, A., and Laing, D. G. (1999). A limit in the processing of components in odour mixtures. Perception 28, 395-404.

Kay, L., and Sherman, S. (2007). An argument for an olfactory thalamus. Trends Neurosci. 30, 47-53.

Kay, L. M., and Laurent, G. (1999). Odor- and context-dependent modulation of mitral cell activity in behaving rats. Nat. Neurosci. 2, 1003-1009.

Kepecs, A., Naoshige, U., and Mainen, Z. F. (2006). The sniff as a unit of olfactory processing. Chem. Senses 31, 167-179.

Khan, R. M., Luk, C. H., Flinker, A., Aggarwal, A., Lapid, H., Haddad,
R., and Sobel, N. (2007). Predicting odor pleasantness from odorant structure: pleasantness as a reflection of the physical world. J. Neurosci. 27, 10015-10023.

Knaapila, A., Tuorila, H., Kyvik, K. O., Wright, M. J., Keskitalo, K., Hansen, J., Kaprio, J., Perola, M., and Silventoinen, K. (2008). Self-ratings of olfactory function reflect odor annoyance rather than olfactory acuity. Laryngoscope 118, 2212-2217.

Krauel, K., Pause, B. M., Sojka, B., Schott, P., and Ferst, R. (1998). Attentional modulation of central odor processing. Chem. Senses 23, 423-432.

Laing, D. G. (1983). Natural sniffing gives optimum odour perception for humans. Perception 12, 99-117.

Laing, D. G., and Glemarec, A. (1992). Selective attention and the perceptual analysis of odor mixtures. Physiol. Behav. 52, 1047-1053.

Lamme, V. A. F. (2003). Why visual attention and awareness are different. Trends Cogn. Sci. (Regul. Ed.) 7, 12-18.

Li, W., Howard, J. D., Parrish, T. B., and Gottfried, J. A. (2008). Aversive learning enhances perceptual and cortical discrimination of indiscriminable odor cues. Science 319, 1842-1845.

Li, W., Lopez, L., Osher, J., Howard, J. D., Parrish, T. B., and Gottfried, J. A. (2010). Right orbitofrontal cortex mediates conscious olfactory perception. Psychol. Sci. 21, 1454-1463.

Liu, T. (2003). Cortical mechanisms of feature-based attentional control. Cereb. Cortex 13, 1334-1343.

Lledo, P.-M., Gheusi, G., and Vincent, J.-D. (2005). Information processing in the mammalian olfactory system. Physiol. Rev. 85, 281-317.

Lorente De No, R. (1938). "The cerebral cortex: architecture, intracortical connections and motor projections," in Physiology of the Nervous System, ed. J. F. Fulton (London: Oxford University Press), 291-325.

Lorig, T. (1992). "Cognitive and 'noncognitive' effects of odor exposure: electrophysiological and behavioral evidence," in The Psychology and Biology of Perfume, eds S. Van Toller and G. Dodd (Amsterdam: Elsevier), 161-173.

Lycan, W. G. (2000). "The slighting of smell," in Of Minds and Molecules: New Philosophical Perspectives on Chemistry, eds N. Bhushan and S. Rosenfeld (Oxford: Oxford University Press), 273-289. 
Macdonald, J. S. P., and Lavie, N. (2008). Load induced blindness. $J$. Exp. Psychol. Hum. Percept. Perform. 34, 1078-1091.

Mack, A., and Rock, I. (1998). Inattentional Blindness. Cambridge: MIT Press.

Mainland, J., and Sobel, N. (2006). The sniff is part of the olfactory percept. Chem. Senses 31, 181-196.

Masago, R., Shimomura, Y., Iwanaga, K., and Katsuura, T. (2001). The effects of hedonic properties of odors and attentional modulation on the olfactory event-related potentials. J. Physiol. Anthropol. Appl. Human Sci. 20, 7-13.

Mcalonan, K., Cavanaugh, J., and Wurtz, R. H. (2008). Guarding the gateway to cortex with attention in visual thalamus. Nature 456, 391-394.

Mccormick, D. A., and Bal, T. (1994). Sensory gating mechanisms of the thalamus. Curr. Opin. Neurobiol. 4, 550-556.

Metzinger, T. (ed.). (2000). Neural Correlates of Consciousness: Empirical and Conceptual Questions. Cambridge: MIT Press.

Mole, C. (2008). Attention and consciousness. J. Conscious. Stud. 15, 86-104.

Morsella, E., Krieger, S. C., and Bargh, J. A. (2010). Minimal neuroanatomy for a conscious brain: homing in on the networks constituting consciousness. Neural Netw. 23, 14-15.

Muller, M. M. (2006). Feature-selective attention enhances color signals in early visual areas of the human brain. Proc. Natl. Acad. Sci. U.S.A. 103, 14250-14254.

Murakami, M., Kashiwadani, H., Kirino, Y., and Mori, K. (2005). State-dependent sensory gating in olfactory cortex. Neuron 46, 285-296.

Nakanishi, S. (1995). Second-order neurones and receptor mechanisms in visual- and olfactory-information processing. Trends Neurosci. 18, 359-364.

Neville, K. R., and Haberly, L. B. (2004). "Olfactory cortex," in The Synaptic Organization of the Brain, 5th Edn, ed. G. M. Shepherd (New York: Oxford University Press), 415-454.

Newman, J. (1995). Thalamic contributions to attention and consciousness. Conscious. Cogn. 4, 172-193.

O'Doherty, J., Rolls, E. T., Francis, S., Bowtell, R., Mcglone, F., Kobal, G., Renner, B., and Ahne, G. (2000). Sensory-specific satiety related olfactory activation of the human orbitofrontal cortex. Neuroreport 11, 893-897.

Pause, B. M., Sojka, B., and Ferstl, R. (1997). Central processing of odor concentration is a temporal phenomenon as revealed by chemosensory event-related potentials (CSERP). Chem. Senses 22, 9-26.

Plailly, J., Howard, J. D., Gitelman, D. R., and Gottfried, J. A. (2008). Attention to odor modulates thalamocortical connectivity in the human brain. $J$. Neurosci. 28, 5257-5267.

Porter, J., Anand, T., Johnson, B., Khan, R. M., and Sobel, N. (2005). Brain mechanisms for extracting spatial information from smell. Neuron 47, 581-592.

Porter, J., Craven, B., Khan, R. M., Chang, S.-J., Kang, I., Judkewicz, B., Volpe, J., Settles, G., and Sobel, N. (2006). Mechanisms of scenttracking in humans. Nat. Neurosci. $10,27-29$.

Posner, M. I. (1994). Attention the mechanism of consciousness. Proc. Natl. Acad. Sci. U.S.A. 91, 7398-7403.

Potter, H., and Butters, N. (1980). An assessment of olfactory deficits in patients with damage to prefrontal cortex. Neuropsychologia 18, 621-628.

Price, J. L. (1985). Beyond the primary olfactory cortex: olfactory-related areas in the neocortex, thalamus and hypothalamus. Chem. Senses 10, 239-258.

Price, J. L., Carmichael, S. T., Carnes, K. M., Clugnet, M.-C., Kuroda, M., and Ray, J. P. (1991). "Olfactory input to the prefrontal cortex," in Olfaction: A Model for Computational Neuroscience, eds J. L. Davis and H. Eichenbaum (Cambridge: MIT Press), 101-120.

Price, J. L., and Slotnick, B. M. (1983). Dual olfactory representation in the rat thalamus: an anatomical and electrophysiological study. J. Comp. Neurol. 215, 63-77.

Prinz, J. J. (2010). "When is perception conscious?" in Perceiving the World: New Essays on Perception, ed. B. Nanay (New York: Oxford University Press), 310-332.

Radil, T., and Wysocki, C. J. (1998). Spatiotemporal masking in pure olfaction. Ann. N. Y. Acad. Sci. 855 641-644.

Raymond, J. E., Shapiro, K. L., and Arnell, K. M. (1992). Temporary suppression of visual processing in an RSVP task: an attentional blink? J. Exp. Psychol. Hum. Percept. Perform. $18,849-860$.
Rousseaux, M., Muller, P., Gahide, I., Mottin, Y., and Romon, M. (1996). Disorders of smell, taste, and food intake in a patient with a dorsomedial thalamic infarct. Stroke 27 2328-2330.

Sabri, M., Radnovich, A. J., Li, T. Q., and Kareken, D. A. (2005). Neural correlates of olfactory change detection. Neuroimage 25, 969-974.

Sela, L., Sacher, Y., Serfaty, C., Yeshurun, Y., Soroker, N., and Sobel, N. (2009). Spared and impaired olfactory abilities after thalamic lesions. J. Neurosci. 29, 12059-12069.

Sela, L., and Sobel, N. (2010). Human olfaction: a constant state of changeblindness. Exp. Brain Res. 205, 13-29.

Shepherd, G. M. (2007). Perspectives on olfactory processing, conscious perception, and orbitofrontal cortex. Ann. N. Y. Acad. Sci. 1121, 87-101.

Shepherd, G. M., Chen, W. R., and Greer, C. A. (2004). "Olfactory Bulb," in The Synaptic Organization of the Brain, 5th Edn, ed. G. M. Shepherd (New York: Oxford University Press), 165-216.

Sherman, S. M. (2001). Tonic and burst firing: dual modes of thalamocortical relay. Trends Neurosci. 24 122-126.

Simons, D., and Rensink, R. (2005). Change blindness: past, present, and future. Trends Cogn. Sci. (Regul. Ed.) 9, 16-20.

Small, D. M., Voss, J., Mak, Y. E. Simmons, K. B., Parrish, T., and Gitelman, D. (2004). Experiencedependent neural integration of taste and smell in the human brain. J. Neurophysiol. 92, 1892-1903.

Smythies, J. (1997). The functional neuroanatomy of awareness: with a focus on the role of various anatomical systems in the control of intermodal attention. Conscious. Cogn. 6 , 455-481.

Spence, C., and Driver, J. (1997). On measuring selective attention to an expected sensory modality. Percept. Psychophys. 59, 389-403.

Spence, C., Kettenmann, B., Kobal, G., and Mcglone, F. P. (2000). Selective attention to the chemosensory modality. Percept. Psychophys. 62 , 1265-1271.

Spence, C., Kettenmann, B., Kobal, G., and Mcglone, F. P. (2001a). Shared attentional resources for processing visual and chemosensory information. Q. J. Exp. Psychol. Hum. Exp. Psychol. 54, 775-783.
Spence, C., Mcglone, F., Kettenmann, B., and Kobal, G. (2001b). Attention to olfaction - a psychophysical investigation. Exp. Brain Res. 138, 432-437.

Stevenson, R. (2009). Phenomenal and access consciousness in olfaction. Conscious. Cogn. 18, 1004-1017.

Stevenson, R. J., and Boakes, R. A. (2003). A mnemonic theory of odor perception. Psychol. Rev. 110, 340-364.

Stevenson, R. J., and Wilson, D. A. (2007). Odour perception: an object-recognition approach. Perception 36, 1821-1833.

Takagia, S. F. (1986). Studies on the olfactory nervous system of the old world monkey. Prog. Neurobiol. 27, 195-250.

Tham, W. W. P., Stevenson, R. J., and Miller, L. A. (2011a). The impact of mediodorsal thalamic lesions on olfactory attention and flavor perception. Brain Cogn. 77, 71-79.

Tham, W. W. P., Stevenson, R. J., and Miller, L. A. (2011b). The role of the medio dorsal thalamic nucleus in human olfaction. Neurocase 17, 148-159.

Tononi, G., and Koch, C. (2008). The neural correlates of consciousness: an update. Ann. N. Y. Acad. Sci. 1124, 239-261.

Van Boxtel, J. J. A., Tsuchiya, N., and Koch, C. (2010). Consciousness and attention: on sufficiency and necessity. Front. Psychol. 1:217. doi:10.3389/fpsyg.2010.00217

Veldhuizen, M. G., and Small, D. M. (2011). Modality-specific neural effects of selective attention to taste and odor. Chem. Senses 36, 747-760.

Verhagen, J. V., Wesson, D. W., Netoff, T. I., White, J. A., and andWachowiak, M. (2007). Sniffing controls an adaptive filter of sensory input to the olfactory bulb. Nat. Neurosci. 10, 631-639.

Wig, G. S., Schlaggar, B. L., and Petersen, S. E. (2011). Concepts and principles in the analysis of brain networks. Ann. N. Y. Acad. Sci. 1224, 126-146.

Wilson, D. A. (2010). Single-unit activity in piriform cortex during slowwave state is shaped by recent odor experience. J. Neurosci. 30 1760-1765.

Wojciulik, E., Kanwisher, N., and Driver, J. (1998). Covert visual attention modulates face-specific activity in the human fusiform gyrus: fMRI study. J. Neurophysiol. 79, 1574-1578.

Yarita, H., Iino, M., Tanabe, T., Kogure, S., and Takagi, S. F. (1980). 
Transthalamic olfactory pathway to orbitofrontal cortex in the monkey. J. Neurophysiol. 43, 69-85.

Yeshurun, Y., and Sobel, N. (2010). An odor is not worth a thousand words: from multidimensional odors to unidimensional odor objects. Annu. Rev. Psychol. 61, 219-241.

Zald, D. H., and Rauch, S. (2008). The Orbitofrontal Cortex. Oxford: Oxford University Press.
Zelano, C., Bensafi, M., Porter, J., Mainland, J., Johnson, B., Bremner, E., Telles, C., Khan, R., and Sobel, N. (2005). Attentional modulation in human primary olfactory cortex. Nat. Neurosci. 8, 114-120.

Conflict of Interest Statement: The author declares that the research was conducted in the absence of any commercial or financial relationships that could be construed as a potential conflict of interest.

Received: 03 October 2011; paper pending published: 31 October 2011; accepted: 30 November 2011; published online: 16 December 2011.

Citation: Keller A (2011) Attention and olfactory consciousness. Front. Psychology 2:380. doi: 10.3389/fpsyg.2011.00380
This article was submitted to Frontiers in Consciousness Research, a specialty of Frontiers in Psychology.

Copyright () 2011 Keller. This is an open-access article distributed under the terms of the Creative Commons Attribution Non Commercial License, which permits non-commercial use, distribution, and reproduction in other forums, provided the original authors and source are credited. 